University of Wollongong

Research Online

Australian Institute for Innovative Materials -

Papers

Australian Institute for Innovative Materials

$1-1-2011$

Critical property in relaxor-PbTiO3 single crystals - Shear piezoelectric response

Fei Li

Xi'an Jiaotong University

Shujun Zhang

The Pennsylvania State University, shujun@uow.edu.au

Zhuo Xu

Xi'an Jiaotong University

Xiaoyong Wei

Xi'an Jiaotong University

Thomas R. Shrout

The Pennsylvania State University

Follow this and additional works at: https://ro.uow.edu.au/aiimpapers

Part of the Engineering Commons, and the Physical Sciences and Mathematics Commons

Research Online is the open access institutional repository for the University of Wollongong. For further information contact the UOW Library: research-pubs@uow.edu.au 


\title{
Critical property in relaxor-PbTiO3 single crystals - Shear piezoelectric response
}

\begin{abstract}
The shear piezoelectric behavior in relaxor-PbTiO 3 (PT) single crystals is investigated with regard to the crystal phase. High levels of shear piezoelectric activity, d 15 or d $24>2000$ pC N-1, are observed for single-domain rhombohedral $(R)$, orthorhombic $(0)$, and tetragonal $(T)$ relaxor-PT crystals. The high piezoelectric response is attributed to a flattening of the Gibbs free energy at compositions close to the morphotropic phase boundaries, where polarization rotation is easily accomplished by applying a perpendicular electric field. The shear piezoelectric behavior of perovskite ferroelectric crystals is discussed with respect to ferroelectric-ferroelectric phase transitions and the dc bias field using a phenomenological approach. The relationship between the single-domain shear piezoelectric response and piezoelectric activities in domain-engineered configurations is also given in this paper. From an application viewpoint, the temperature and ac-field drive stability for shear piezoelectric responses are investigated. A temperature-independent shear piezoelectric response $\left(\mathrm{d} 24\right.$, in the range of $-50^{\circ} \mathrm{C}$ to the $0-T$ phase-transition temperature) is thermodynamically expected and experimentally confirmed in orthorhombic relaxor-PT crystals; a relatively high ac-field drive stability $(5 \mathrm{kV} \mathrm{cm} \mathrm{-1)} \mathrm{is} \mathrm{obtained} \mathrm{in}$ manganese-modified relaxor-PT crystals. For all thickness shear vibration modes, the mechanical quality factor Qs are less than 50, corresponding to the facilitated polarization rotation. The shear piezoelectric behavior in relaxor-PbTiO 3 (PT) single crystals is systematically investigated with regard to the crystal phase. High levels of shear piezoelectric activity, d 15 or d $24>2000$ pC N -1, are observed for singledomain rhombohedral $(R)$, orthorhombic $(0)$, and tetragonal $(T)$ relaxor-PT crystals. These properties are investigated with respect to the temperature and electric field. The results demonstrate that the singledomain relaxor-PbTiO 3 crystals are promising materials for high-performance transducers. Copyright 2011 WILEY-VCH Verlag GmbH \& Co. KGaA, Weinheim.
\end{abstract}

\section{Keywords}

single, crystals, shear, piezoelectric, critical, response, relaxor, property, pbtio3

Disciplines

Engineering | Physical Sciences and Mathematics

\section{Publication Details}

Li, F., Zhang, S., Xu, Z., Wei, X. \& Shrout, T. R. (2011). Critical property in relaxor-PbTiO3 single crystals Shear piezoelectric response. Advanced Functional Materials, 21 (11), 2118-2128. 


\title{
Critical Property in Relaxor-PbTiO 3 Single Crystals - Shear Piezoelectric Response
}

\author{
Fei Li, Shujun Zhang,* Zhuo Xu, Xiaoyong Wei, and Thomas R. Shrout
}

The shear piezoelectric behavior in relaxor- $\mathrm{PbTiO}_{3}$ (PT) single crystals is investigated with regard to the crystal phase. High levels of shear piezoelectric activity, $d_{15}$ or $d_{24}>2000 \mathrm{pC} \mathrm{N} \mathrm{N}^{-1}$, are observed for single-domain rhombohedral ( $R$ ), orthorhombic (O), and tetragonal (T) relaxor-PT crystals. The high piezoelectric response is attributed to a flattening of the Gibbs free energy at compositions close to the morphotropic phase boundaries, where polarization rotation is easily accomplished by applying a perpendicular electric field. The shear piezoelectric behavior of perovskite ferroelectric crystals is discussed with respect to ferroelectric-ferroelectric phase transitions and the dc bias field using a phenomenological approach. The relationship between the single-domain shear piezoelectric response and piezoelectric activities in domain-engineered configurations is also given in this paper. From an application viewpoint, the temperature and ac-field drive stability for shear piezoelectric responses are investigated. A temperature-independent shear piezoelectric response $\left(d_{24}\right.$, in the range of $-50^{\circ} \mathrm{C}$ to the O-T phase-transition temperature) is thermodynamically expected and experimentally confirmed in orthorhombic relaxor-PT crystals; a relatively high ac-field drive stability $\left(5 \mathrm{kV} \mathrm{cm}{ }^{-1}\right)$ is obtained in manganese-modified relaxor-PT crystals. For all thickness shear vibration modes, the mechanical quality factor $Q s$ are less than 50 , corresponding to the facilitated polarization rotation.

\section{Introduction}

Relaxor- $\mathrm{PbTiO}_{3}$ (PT) based ferroelectric single crystals, such as $\quad(1-x) \mathrm{Pb}\left(\mathrm{Zn}_{1 / 3} \mathrm{Nb}_{2 / 3}\right) \mathrm{O}_{3}-x \mathrm{PbTiO}_{3} \quad(\mathrm{PZN}-\mathrm{PT})$ and $(1-x)$ $\mathrm{Pb}\left(\mathrm{Mg}_{1 / 3} \mathrm{Nb}_{2 / 3}\right) \mathrm{O}_{3}-\mathrm{PbTiO}_{3}$ (PMN-PT) have attracted considerable interest over the last decade because of their superior piezoelectric properties compared to commercial piezoelectric ceramics, including (1-x) $\mathrm{PbZrO}_{3}-x \mathrm{PbTiO}_{3}$ (PZT). The ultrahigh piezoelectric response $\left(d_{33}>1500 \mathrm{pC} \mathrm{N}^{-1}, k_{33}>95 \%\right)$

\section{F. Li}

Electronic Materials Research Laboratory

Xi'an Jiaotong University

Xi'an 710049, P. R. China

Fei Li, Prof. S. J. Zhang, Prof. T. R. Shrout

Materials Research Institute

Pennsylvania State University

University park, PA 16802, USA

E-mail: soz1@psu.edu

Prof. Z. Xu, Prof. X. Y. Wei

Electronic Materials Research Laboratory

Xi'an Jiaotong University

Xi'an 710049, P. R. China

DOI: $10.1002 / \mathrm{adfm} .201002711$ was found to be along the [001] crystallographic direction for compositions lying in proximity to a morphotropic phase boundary (MPB). ${ }^{[1-7]}$ In contrast to polycrystalline piezoelectric ceramics, high piezoelectric activities in relaxor-PT based crystals is believed to be intrinsic in nature. ${ }^{[7]}$ Two intrinsic-based mechanisms have been proposed to explain the origin of the high piezoelectric response. The first one is "polarization rotation", ${ }^{[8-10]}$ where the high piezoelectric response is attributed to an 'ease' in polarization rotation. It is believed that the monoclinic (M) phase ${ }^{[10-16]}$ acts as a bridge between the morphotropic phases [rhombohedral (R) and tetragonal (T)], facilitating the polarization rotation..$^{[10-12,17,18]}$ For the second intrinsic mechanism, the high piezoelectric response is attributed to the high level of shear piezoelectric response in the single-domain state, ${ }^{[19]}$ which can greatly contribute to the longitudinal piezoelectric response in [001] and [011] domain-engineered crystals. As analyzed by Damjanovic et al. ${ }^{[20-23]}$ the high shear piezoelectric activity is related to a flattening of the free energy profile (instability of free energy) near the MPB. Actually, the high shear piezoelectric response is directly related to the "ease" in polarization rotation, as has been revealed before. ${ }^{[23]}$

From an application viewpoint, the investigation of relaxorPT crystals has been focused on crystal growth technology and usage temperature range. Relaxor-PT based crystals with large size, homogeneous composition, and low cost are desirable for various applications. To date, the PMN-PT and $\mathrm{Pb}\left(\mathrm{In}_{1 / 2} \mathrm{Nb}_{1 / 2}\right)$ $\mathrm{O}_{3}-\mathrm{Pb}\left(\mathrm{Mg}_{1 / 3} \mathrm{Nb}_{2 / 3}\right) \mathrm{O}_{3}-\mathrm{PbTiO}_{3}$ (PIN-PMN-PT) crystals can be grown with relatively large size (3-inch in diameter) using the modified Bridgman method. ${ }^{[24,25]}$ A continuous feeding technique has also been reported to grow relaxor-PT based crystals with uniform composition and properties. ${ }^{[26]}$ For commercial PMN-PT crystals, the temperature usage range is limited by the ferroelectric-ferroelectric phase-transition temperature $T_{R-T}$ to $<95{ }^{\circ} \mathrm{C} .{ }^{[6]}$ In recent years, ternary PIN-PMN-PT crystals have been grown and investigated, showing a broadened temperature usage range $\left(T_{\mathrm{R}-\mathrm{T}}\right.$ around $\left.120^{\circ} \mathrm{C}\right) \cdot{ }^{[27-32]} \mathrm{To}$ further enhance the usage temperature range for relaxor-PT crystals, efforts have been put into growing tetragonal PIN-PMN-PT crystals, in which a broadened temperature usage range $\left(>200^{\circ} \mathrm{C}\right)$ together with good electromechanical coupling $k_{33}$ (ca. $84 \%$ ) have been 
observed, as no ferroelectric-ferroelectric phase transition occurs above room temperature. ${ }^{[33]}$

Until now, piezoelectric investigations for relaxor-PT based crystals have been mainly focused on their longitudinal behavior, whereas the transverse (shear) piezoelectric and dielectric properties of relaxor-PT crystals have not been extensively studied. ${ }^{[34-38]}$ In contrast to the longitudinal mode, the transverse (shear) piezoelectric/dielectric response is much higher in single-domain crystals, ${ }^{[34-39]}$ making relaxor-PT based crystals good candidates for various shear-mode applications, such as vector sensors, non-destructive evaluation (NDE) transducers, and low-frequency sonar. Furthermore, the shear piezoelectric activity of the single-domain state is believed to be a critical factor for the origin of the high longitudinal piezoelectric response in the domain-engineered state. Thus, it is desirable to systematically study the shear properties in relaxor-PT crystals.

In this paper, the present understanding of the piezoelectric shear response of relaxor-PT based crystals is first discussed. The shear piezoelectric behavior is then analyzed with respect to the polarization rotation process, including the temperature and composition dependence of the shear properties, the contribution of shear piezoelectric to longitudinal piezoelectric properties in domain-engineered crystals, and shear piezoelectricity in domain-engineered states. From an application viewpoint, the temperature and ac-field drive stability, and the mechanical quality factor $Q$ of the shear vibration modes are discussed. Approaches to improve the temperature and ac-field drive stability are also proposed.

\section{Shear Piezoelectric Behavior in Relaxor-PT Based Ferroelectric Crystals}

\subsection{Shear Piezoelectric Behavior in Single-Domain Configuration}

The shear piezoelectric properties in relaxor-PT based crystals were studied for various ferroelectric phases, including the rhombohedral $(\mathrm{R})$, orthorhombic $(\mathrm{O})$, and tetragonal $(\mathrm{T})$ phases. Figure 1 shows the schematic phase diagram, showing the $\mathrm{R}, \mathrm{O}$, and $\mathrm{T}$ ferroelectric phases and paraelectric phase $(\mathrm{C})$. (The monoclinic $\mathrm{M}_{\mathrm{C}}$ phase was found to be only slightly distorted from the orthorhombic phase. Thus, after poling along the [011] direction, it was proper to consider the $\mathrm{M}_{\mathrm{C}}$ phase as a

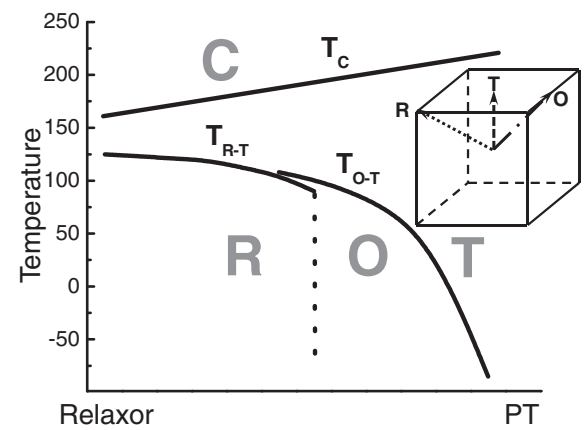

Figure 1. Schematic phase diagram for relaxor-PT based crystals, where $\mathrm{R}, \mathrm{O}$, and $\mathrm{T}$ represent rhombohedral, orthorhombic/monoclinic $\left(\mathrm{M}_{\mathrm{C}}\right)$, and tetragonal phase regions. ${ }^{[14,15]}$ The spontaneous polar directions of $\mathrm{R}, \mathrm{O}$, and $\mathrm{T}$ crystals are depicted in a pseudo-cubic lattice (measured results of the PIN-PMN-PT system were taken as an example).

quasi-orthorhombic phase for analysis). ${ }^{[14,15]}$ The spontaneous polarization vectors of the rhombohedral, orthorhombic, and tetragonal crystals lie along the $[111]_{\mathrm{C}},[011]_{\mathrm{C}}$, and $[001]_{\mathrm{C}}$ crystallographic directions, respectively, as depicted in Figure 1. For the rhombohedral, orthorhombic, and tetragonal crystals, by poling along their respective polar directions, the symmetry is $3 \mathrm{~m}$ with one independent shear piezoelectric coefficient $\left(d_{15}=\right.$ $\left.d_{24}\right)$, mm2 with two independent shear coefficients $\left(d_{15} \neq d_{24}\right)$, and $4 \mathrm{~mm}$ with one independent shear coefficient $\left(d_{15}=d_{24}\right)$, respectively. Table 1 summarizes the shear piezoelectric coefficients of the rhombohedral, orthorhombic, and tetragonal phases for PIN-PMN-PT crystals with compositions around the MPB. ${ }^{[33,40]}$ High shear piezoelectric coefficients $\left(>2000 \mathrm{pC} \mathrm{N}^{-1}\right)$ and electromechanical coupling factors $(>85 \%)$ were observed at room temperature for all single-domain crystals, including rhombohedral "1R", orthorhombic "1O", and tetragonal "1T" domain configuration (where " 1 " indicates the single-domain configuration). ${ }^{[41]}$

By applying an electric field perpendicular to the polar direction of ferroelectric crystals, the polarization in these crystals will rotate in order to minimize the free energy, ${ }^{[42]}$ leading to a shear deformation of the lattice, as depicted in Figure 2. For single-domain relaxor-PT crystals, therefore, the shear piezoelectric response is associated with these polarization rotation processes. ${ }^{[22]}$ As analyzed in the following, the polarization rotation is easier for compositions closer to the MPB, leading to a high level of shear piezoelectric activity.

Table 1. Electromechanical shear properties for PIN-PMN-PT crystals with rhombohedral, orthorhombic, and tetragonal phases.

\begin{tabular}{|c|c|c|c|c|}
\hline Phase & Rhombohedral & & Orthorhombic & Tetragonal \\
\hline Domain Configuration & $1 \mathrm{R}$ & 10 & 10 & $1 \mathrm{~T}$ \\
\hline Symmetry & $3 m$ & $\mathrm{~mm} 2$ & $\mathrm{~mm} 2$ & $4 \mathrm{~mm}$ \\
\hline \multirow[t]{4}{*}{ Properties } & $d_{15}>3000 \mathrm{pC} \mathrm{N} \mathrm{N}^{-1}$ & $d_{15}>3000 \mathrm{pC} \mathrm{N} \mathrm{N}^{-1}$ & $d_{24} \sim 2000 \mathrm{pC} \mathrm{N} \mathrm{N}^{-1}$ & $d_{15} \sim 2000 \mathrm{pC} \mathrm{N} \mathrm{N}^{-1}$ \\
\hline & $k_{15}>90 \%$ & $k_{15}>90 \%$ & $k_{24}>85 \%$ & $k_{15} \sim 80 \%$ \\
\hline & $\varepsilon_{11}>6000$ & $\varepsilon_{11}>6000$ & $\varepsilon_{22}>15000$ & $\varepsilon_{11}>12000$ \\
\hline & $\mathrm{s}_{55}{ }^{\mathrm{D}} \sim 27 \mathrm{pm}^{2} \mathrm{~N}^{-1}$ & $\mathrm{~S}_{55}{ }^{\mathrm{D}} \sim 26 \mathrm{pm}^{2} \mathrm{~N}^{-1}$ & $\mathrm{~S}_{55}{ }^{\mathrm{D}} \sim 15 \mathrm{pm}^{2} \mathrm{~N}^{-1}$ & $\mathrm{~S}_{55}{ }^{\mathrm{D}} \sim 15 \mathrm{pm}^{2} \mathrm{~N}^{-1}$ \\
\hline
\end{tabular}




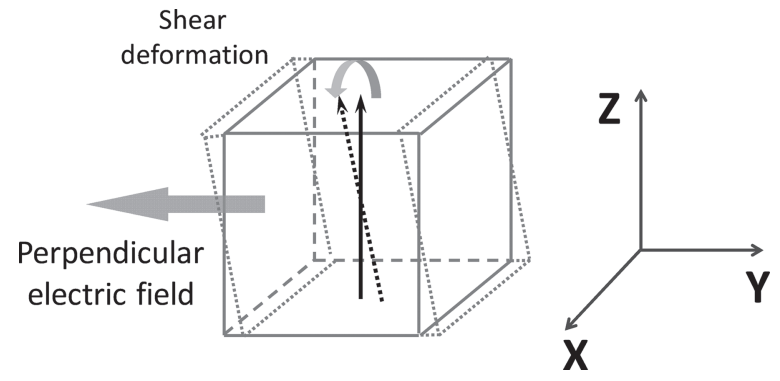

Figure 2. Schematic polarization rotation process and related shear piezoelectric deformation, under perpendicular electric field, whereby the polarizations are represented by arrows.

\subsection{Role of Ferroelectric-Ferroelectric Phase Transitions for Shear Piezoelectric Response}

From a thermodynamic analysis on perovskite ferroelectric crystals, the shear piezoelectric coefficients generally exhibit maximum values around the ferroelectric-ferroelectric phase transitions, no matter if it is induced by the composition or by temperature, because of the flattening of the free-energy profile or the enhanced energy instability around the phase-transition points, ${ }^{[20-23]}$ as depicted in Figure 3. Based on previous measurements and thermodynamic analysis, ${ }^{[20,40,43]}$ the characteristics of shear piezoelectric coefficients in rhombohedral, orthorhombic, and tetragonal crystals can be summarized as follows:

1) In the rhombohedral phase, there is only one independent piezoelectric coefficient $d_{15}$ for the $3 \mathrm{~m}$ symmetry, which increases as the composition/temperature approaches the orthorhombic or tetragonal phase from the rhombohedral side.

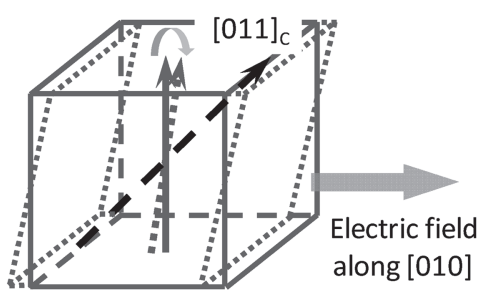

(a)

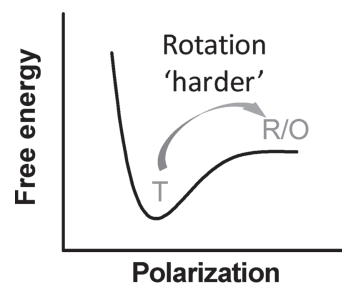

temp./comp. approaches O/R phase

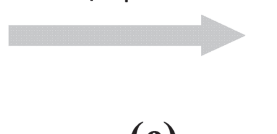

(c)

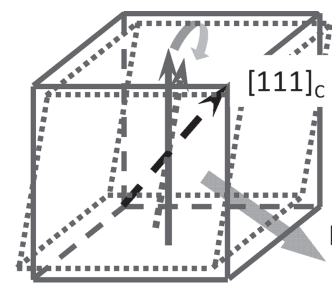

Electric field along [110]

(b)

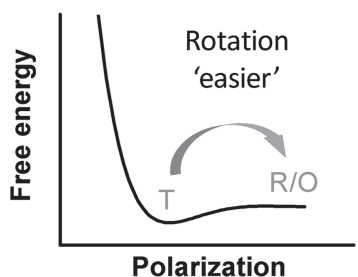

Figure 3. The shear piezoelectric deformations for tetragonal crystals, corresponding to the shear coefficient $d_{15}$. By applying a perpendicular electric field along a) [010] $]_{C}$ and b) [110] $]_{C}$, the polarization rotates to the $[011]_{C}$ (polar direction of orthorhombic phase) and $[111]_{C}$ direction (polar direction of rhombohedral phase), respectively. The subscript " $\mathrm{C}$ " represents the crystallographic axes in a pseudo-cubic lattice.
2) For the tetragonal phase with $4 \mathrm{~mm}$ symmetry, the independent shear piezoelectric coefficient $d_{15}$ will increase as the composition/temperature approaches the rhombohedral or orthorhombic phase.

3) In orthorhombic crystals with $\mathrm{mm} 2$ symmetry, there are two independent shear piezoelectric coefficients, $d_{15}$ and $d_{24}$. As shown in Figure $4,{ }^{[40]}$ because of the different polarization rotation paths, the coefficient $d_{15}$ will increase if the composition/temperature is close to the O-T phase boundary, whereas $d_{24}$ will be enhanced if the composition/temperature is close to the O-R phase boundary/phase-transition temperature.

The above discussion can be used as a basis to analyze the temperature/composition-dependent shear properties for relaxor-PT based crystals with different phases.

\subsection{Shear Piezoelectric Behaviors in Domain-Engineered Crystals}

In contrast to the high level of shear piezoelectric responses in single-domain crystals, both high and low shear coefficients were observed in domain-engineered crystals, for example, in $[001]_{\mathrm{C}}$ poled rhombohedral crystals, $d_{15}{ }^{*}<200 \mathrm{pC} \mathrm{N} \mathrm{N}^{-1 ;[44,45]}$ in $[011]_{\mathrm{C}}$ poled rhombohedral crystals, $d_{15}{ }^{*}>2000 \mathrm{pC} \mathrm{N}^{-1}$ whereas $d_{24}{ }^{*}<200 \mathrm{pC} \mathrm{N}^{-1} \cdot{ }^{[46,47]}$ The properties of multi-domain crystals can be attributed to the single-domain properties and related domain configurations, that is, orientation and structure of the domains. In order to evaluate the difference in single and multidomain configurations, the shear properties of [011] poled rhombohedral crystals were analyzed. ${ }^{[48]}$ Figure 5 shows the domain guration for $[011]_{C}$ poled rhombohedral crystals, where symmetry. The remaining $71^{\circ}$ domains in the " $2 \mathrm{R}$ " domain configuration are designated as domain I and domain II. For the 15-mode, the contributions of domains I and II are equivalent with respect to the perpendicular electric field $E_{1}$, as shown in Figure 6. The polarization rotation of both domains I and II contribute to the shear piezoelectric deformation $S_{5}$, leading to a high shear coefficient $d_{15}{ }^{*}$. However, for the 24-mode, the contribution to the shear deformation $S_{4}$ via polarization rotation of domains I and II are opposed, negating one another, as depicted in Figure 6. ${ }^{[48]}$ Thus, the coefficient $d_{24}{ }^{*}$ is relatively small in $[011]_{\mathrm{C}}$ poled domain-engineered crystals. ${ }^{[4-48]}$ Analogous to the above analysis, a small shear piezoelectric coefficient $d_{15}{ }^{*}$ in $[001]_{\mathrm{C}}$ poled rhombohedral crystals (with "4R" domain configuration) can be expected.

In summary, the high shear piezoelectric behavior of single-domain rhombohedral, orthorhombic, and tetragonal crystals with compositions around the MPB, are a result of flattening of the free-energy profile. The low level of shear piezoelectric responses in domain-engineered crystals can be explained by the negating effect among different domain rotation processes. 


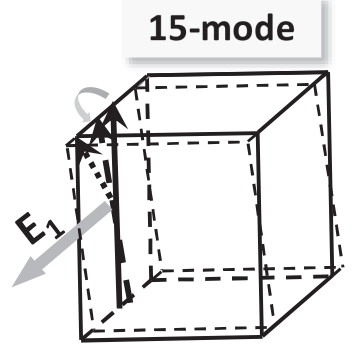

24-mode

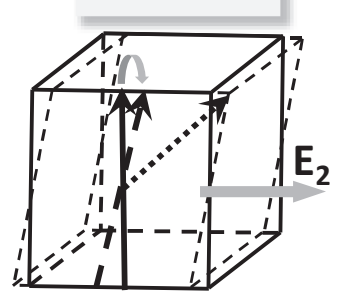

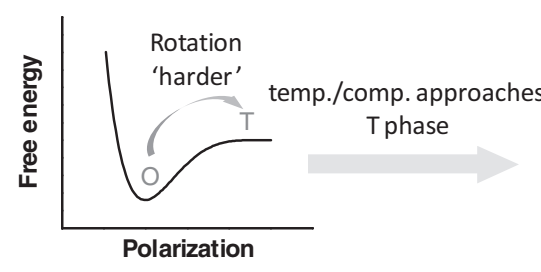

(a)

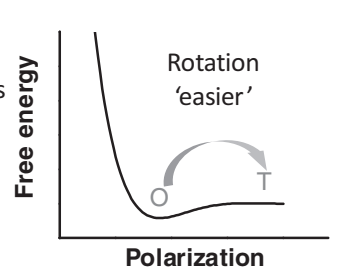

Polarization

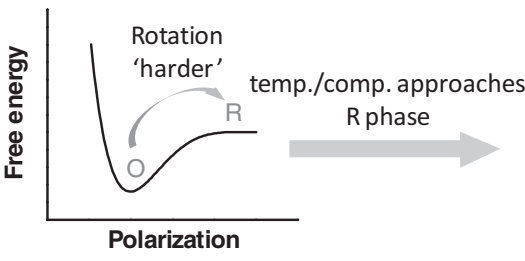

(b)

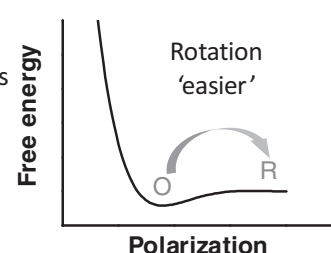

Polarization

Figure 4. Two independent shear piezoelectric responses (15- and 24-mode) and related polarization rotation path in orthorhombic crystals, whereby the solid and dashed arrows represent the polarization rotation process under perpendicular electric field. The electric fields $E_{1}$ and $E_{2}$ are along the $[0-11]_{C}$ and $[100]_{C}$ directions, respectively. The dotted arrows represent the $[001]_{C}$ and $[111]_{C}$ directions in (a) and (b), respectively.

\subsection{Shear Versus Longitudinal Piezoelectric Response}

The high longitudinal piezoelectric response is generally observed along the non-polar direction(s) in relaxor-PT based crystals. ${ }^{[1,49]}$ Taking $[011]_{\mathrm{C}}$ poled tetragonal crystals as an example, the enhanced piezoelectric response in these domainengineered crystals is illustrated in Figure 7. The electric field along the non-polar $[011]_{\mathrm{C}}$ direction can be separated into two parts: $E_{\|}, E_{\perp}$. The $E_{\|}$is along the polar direction and leads to polarization extension; $E \perp$ gives rise to the polarization rotation and consequently the shear deformation of single domains. This shear deformation can contribute to the longitudinal strain along the $[011]_{\mathrm{C}}$ direction. Thus, if the related shear piezoelectric response of the single domains is high, the longitudinal piezoelectric response along the non-polar direction would be greatly enhanced. Based on the single-domain properties, ${ }^{[33,46,50]}$ Figure 8 shows the orientation dependence of the longitudinal piezoelectric coefficient $d_{33}{ }^{*}$ for PIN-PMN-PT crystals. It can be seen that the maximum $d_{33}{ }^{*}$ values are along the non-polar directions for

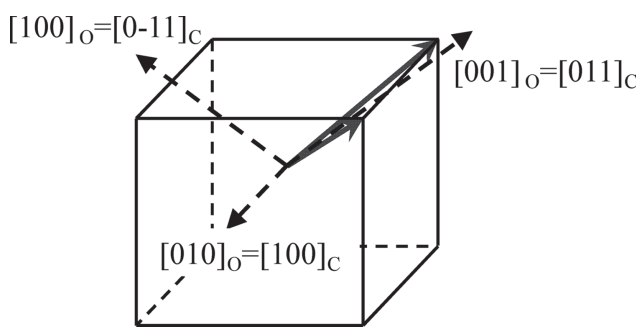

Figure 5. Domain structure of [011] coled rhombohedral crystals, where the solid arrows represent the two different rhombohedral domains. The subscript "O" represents the crystallographic axes in the orthorhombic lattice. all three different phases. As demonstrated, ${ }^{[22,23]}$ if the piezoelectric anisotropy factor, $d_{15} / d_{33}$, is greater than 2 , the maximum longitudinal piezoelectric coefficient will be along the non-polar direction, and this type of crystals is referred to as 'rotator' ferroelectrics. The anisotropic factors and electromechanical properties of various single-domain and domain-engineered configurations are listed in Table 2 (data taken from the literature $\left.^{[1,33,37,39,48-52]}\right)$. For the rhombohedral and orthorhombic crystals, both the longitudinal piezoelectric coefficients and coupling factors are enhanced in domain-engineered configurations, because of the high piezoelectric anisotropy. It is worth noting that although the piezoelectric coefficients are enhanced for domain-engineered tetragonal crystals, the maximum coupling factors $k_{33}{ }^{*}$ were still along the $[001]_{\mathrm{C}}$ polar direction, this is related to the fact that the dielectric anisotropy is greater than the piezoelectric anisotropy factor. ${ }^{[22,49,53]}$

According to the above analysis, the shear piezoelectric response contributes to the longitudinal piezoelectric response in domain-engineered crystals. Furthermore, by Rayleigh analysis of domain-engineered relaxor-PT crystals, the ultrahigh longitudinal piezoelectric response for domain-engineered crystals is mainly attributed to the single-domain shear piezoelectric response. ${ }^{[7,54,55]}$ In addition, the shear piezoelectric responses generally exhibit maximum values around an MPB based on the thermodynamic analysis, thus, the longitudinal piezoelectric response in domain-engineered crystals will be greatly enhanced as the composition approaches an MPB.

\section{Temperature-Dependent Dielectric and Piezoelectric Properties}

Based on the discussion in Section 2.2 and the phase diagram of the relaxor-PT based crystals (Figure 1), the temperature-dependent 



Figure 6. Shear piezoelectric behavior and related polarization rotation processes for $[011]_{\mathrm{C}}$ poled rhombohedral crystals. Reproduced with permission. ${ }^{[48]}$ Copyright 2010, the American Institute of Physics.

shear dielectric and piezoelectric properties were investigated for rhombohedral, orthorhombic, and tetragonal crystals.

For rhombohedral crystals, as presented in Section 2.2, the shear piezoelectric coefficient $d_{15}$ increases with increasing temperature, as the phase approaches the R-T MPB region with increasing temperature. For tetragonal crystals, however, because the T-O ferroelectric phase transition lies below room temperature, the shear piezoelectric coefficient $d_{15}$ is expected to decrease with increasing temperature, moving away from the T-O MPB region. For $[011]_{\mathrm{C}}$ poled orthorhombic crystals, the coefficient $d_{15}$ should increase with increasing temperature, as the O-T phase transition occurs above room temperature. Of particular interest is that a temperature-independent $d_{24}$ can be expected in orthorhombic crystals, because of the existence of a compositionally "vertical" $O-R$ phase boundary in relaxorPT based crystals, as shown in Figure 1, revealing that no $\mathrm{O}-\mathrm{R}$ phase transition occurs in the studied temperature range for orthorhombic relaxor-PT crystals. ${ }^{40]}$

Figure 9 shows the shear piezoelectric behavior for rhombohedral, orthorhombic, and tetragonal PIN-PMN-PT crystals as a function of temperature. The variations of the shear piezoelectric coefficients for PINPMN-PT based crystals are consistent with the above analysis. A temperature-independent shear piezoelectric coefficient $\left(d_{24}\right)$ with an ultrahigh value of $>2000 \mathrm{pC} \mathrm{N}^{-1}$ was observed. ${ }^{[40]}$ The piezoelectric coefficients $d_{15}$ for rhombohedral and orthorhombic crystals were found to increase with increasing temperature and the longitudinal piezoelectric coefficients $d_{33}{ }^{*}$ along the $[001]_{\mathrm{C}}$ direction were expected to increase with increasing temperature (below the $\left.T_{\mathrm{R}-\mathrm{T} / \mathrm{O}-\mathrm{T})}\right)^{[6,29,55-59]}$ On the other hand, for domain-engineered tetragonal crystals (poled along the $[011]_{\mathrm{C}}$ direction), the coefficient $d_{33}{ }^{*}$ was found to decrease with increasing temperature because of the decreased $d_{15}$, as shown in Figure $9 \mathrm{~b} \cdot{ }^{[49]}$ It is worth noting that a temperature-independent longitudinal coefficient $d_{33}{ }^{*}$ can be expected in crystals that are in a single-domain state (poled along the polar direction), where no shear piezoelectric contribution was observed. For example, in $[001]_{\mathrm{C}}$ poled tetragonal PIN-PMN-PT crystals, the $d_{33}{ }^{*}$ maintains the same value up to $200{ }^{\circ} \mathrm{C} .{ }^{[33]}$

The temperature-dependent dielectric permittivity and coupling factors are shown in Figure 10. The temperature characteristics
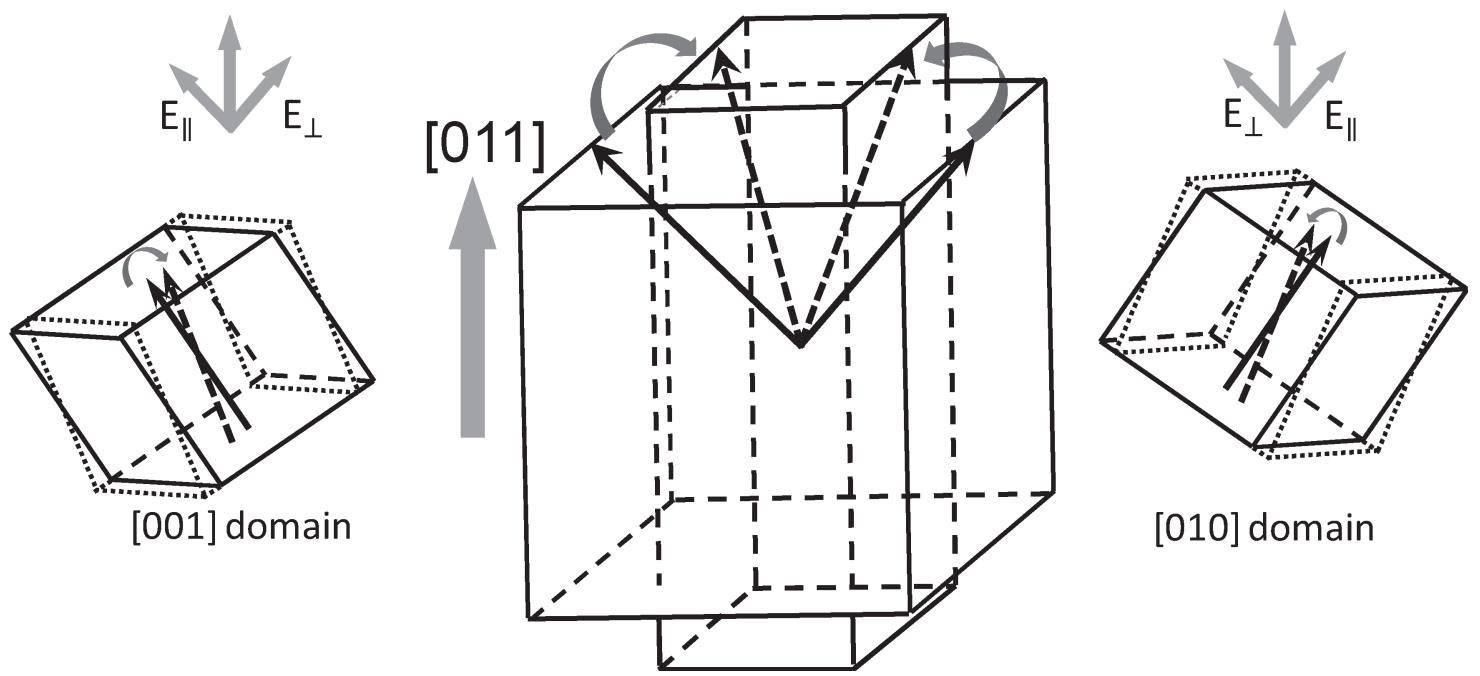

[010] domain

Figure 7. Schematic figure of the piezoelectric deformation in $[011]_{c}$ poled tetragonal crystals. It can be seen that the shear piezoelectric response of single-domain states can contribute to the longitudinal piezoelectric response of domain-engineered crystals. 
Table 2. Piezoelectric and dielectric anisotropies of relaxor-PT based crystals with various phases.

\begin{tabular}{|c|c|c|c|c|c|c|c|c|}
\hline \multirow[t]{2}{*}{ Relaxor-PT crystals } & \multicolumn{7}{|c|}{ Data for single-domain state } & Engineered domain configuration \\
\hline & $\varepsilon_{33} / \varepsilon_{0}$ & $\varepsilon_{11} / \varepsilon_{0}$ & $\varepsilon_{11} / \varepsilon_{33}$ & $d_{33}\left[\mathrm{pC} \mathrm{N} \mathrm{N}^{-1}\right]$ & $d_{15}\left[\mathrm{pC} \mathrm{N} \mathrm{N}^{-1}\right]$ & $d_{15} / d_{33}$ & $k_{33}$ & $k_{33}{ }^{*}$ \\
\hline $\operatorname{PIMNT}(T)^{a)}$ & 1090 & 15000 & 14 & 530 & 2350 & 4 & $84 \%$ & $75 \%$ along [011] \\
\hline $\operatorname{PIMNT}(\mathrm{O})^{\mathrm{b})}$ & 900 & 5400 & 6 & 340 & 3800 & 11 & $82 \%$ & $93 \%$ along [001] \\
\hline $\operatorname{PIMNT}(R)^{c)}$ & 700 & 6300 & 9 & 74 & 2190 & 30 & $36 \%$ & $89 \%$ along [001] \\
\hline PMN-0.42PT(T) $\left.{ }^{d}\right)$ & 660 & 8600 & 13 & 260 & 1300 & 5 & $78 \%$ & 72\% along [011] \\
\hline PMN-0.33PT $(R)^{e)}$ & 640 & 3900 & 6 & 190 & 4100 & 21 & $69 \%$ & $94 \%$ along [001] \\
\hline PZNT-0.12PT $(T)^{f)}$ & 870 & $12000^{a}$ & 14 & 560 & $1450^{\mathrm{a})}$ & 2.5 & $87 \%$ & $62 \%$ along [011] \\
\hline PZNT-0.08PT(R) $)^{\mathrm{g})}$ & 1000 & 16000 & 16 & $90^{\text {a) }}$ & 5000 & 55 & $39 \%$ & $94 \%$ along [001] \\
\hline
\end{tabular}

a-g) References [33,49], [48], [50], [51], [39], [52], and [1,37], respectively.

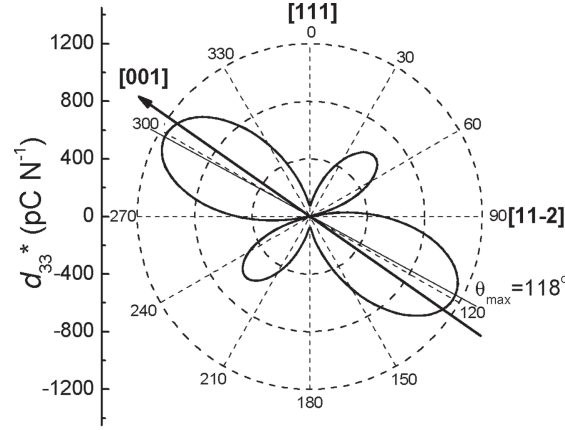

(a)



(b)

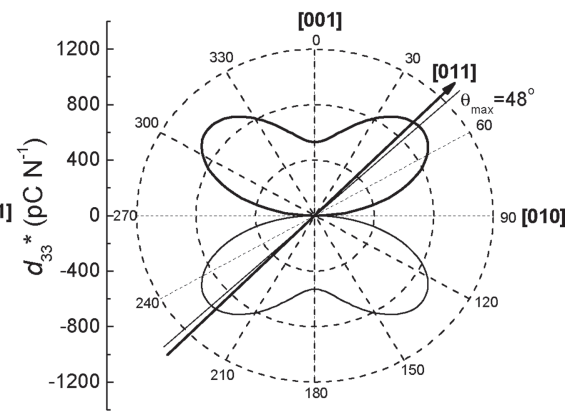

(c)

Figure 8. Orientation dependence of piezoelectric coefficient $d_{33}^{*}$ for single-domain PIN-PMN-PT crystals with a) rhombohedral, b) orthorhombic, and c) tetragonal phases. The respective input data have been reported previously.[50,48,33]

of the dielectric permittivities exhibited a similar trend to that of the piezoelectric coefficients, as predicted by thermodynamic analysis. The dielectric permittivity $\varepsilon_{22} / \varepsilon_{0}$ of orthorhombic crystals was found to be temperature independent, whereas the permittivity $\varepsilon_{11} / \varepsilon_{0}$ of rhombohedral, orthorhombic, and tetragonal crystals showed large variations with temperature.
Because the dielectric and piezoelectric responses to temperature showed similar trends, the electromechanical coupling factors $\left(k \propto d / \varepsilon^{1 / 2}\right)$ were relatively stable with respect to temperature, as shown in Figure 10b. The shear coupling factor $k_{15}$ of a tetragonal PIN-PMN-PT crystal was found to decrease by about $7 \%$ upon raising the temperature from $-50{ }^{\circ} \mathrm{C}$ to $120^{\circ} \mathrm{C}$,

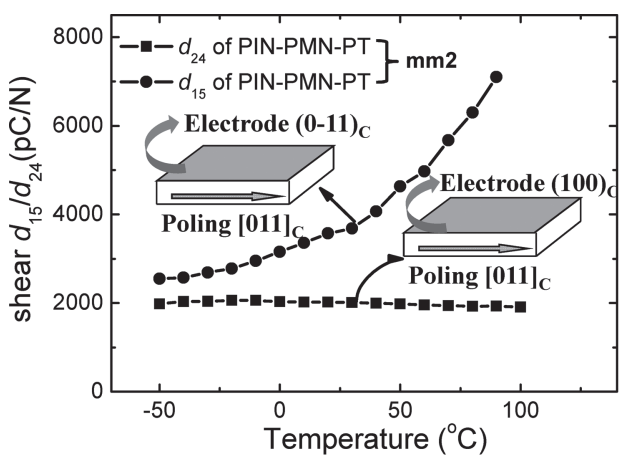

(a)

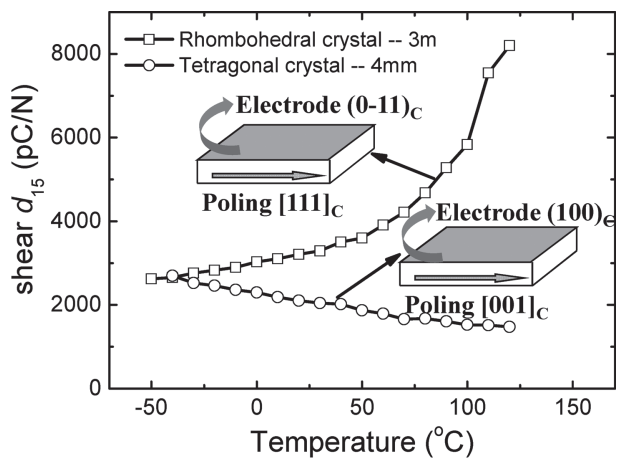

(b)

Figure 9. The temperature dependence of shear piezoelectric coefficients for a) orthorhombic PIN-PMN-PT crystals; b) rhombohedral and tetragonal PIN-PMN-PT crystals. Reproduced with permission. ${ }^{[40]}$ Copyright 2010, the American Institute of Physics. 


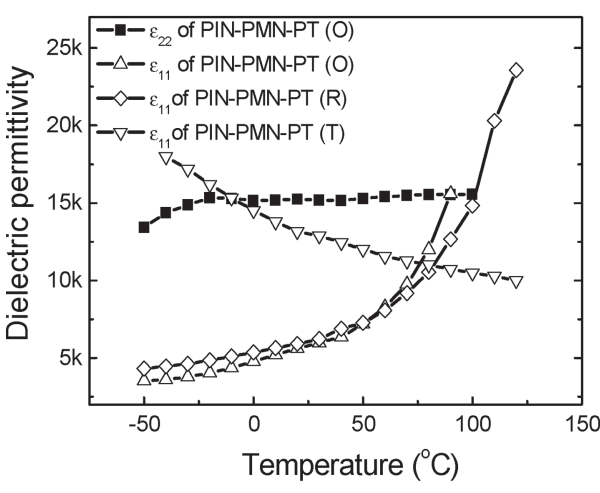

(a)

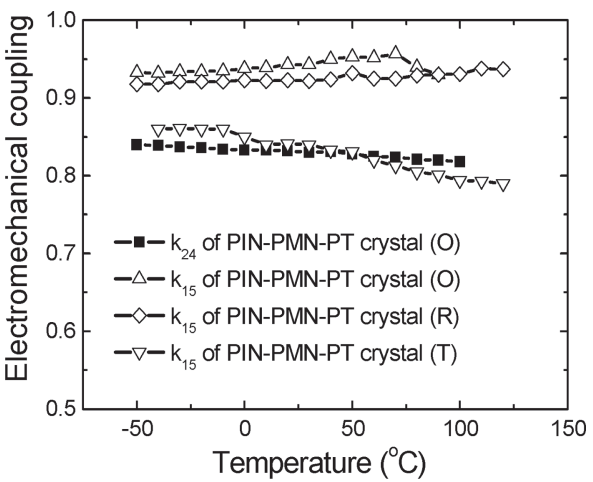

(b)

Figure 10. The temperature dependence of a) transverse dielectric permittivity and b) shear electromechanical couplings for PIN-PMN-PT crystals.

whereas the variation of other shear coupling factors was less than $3 \%$.

\section{Electric-Field-Dependent Piezoelectric Properties}

It is desirable to understand the piezoelectric behavior of relaxor-PT single crystals under a high electric field, including a dc bias field or ac drive field, for potential high-power applications, where the field stability needs to be demonstrated.

\subsection{Shear Piezoelectric Response with Respect to a dc Bias Field}

Under a dc bias field, the free energy of perovskite systems can be expressed as follows: ${ }^{[43]}$

$$
\begin{aligned}
\Delta G= & \alpha_{1}\left(p_{1}^{2}+p_{2}^{2}+p_{3}^{2}\right)+\alpha_{11}\left(p_{1}^{4}+p_{2}^{4}+p_{3}^{4}\right) \\
& +\alpha_{12}\left(p_{1}^{2} p_{2}^{2}+p_{2}^{2} p_{3}^{2}+p_{3}^{2} p_{1}^{2}\right) \\
& +\alpha_{111}\left(p_{1}^{6}+p_{2}^{6}+p_{3}^{6}\right) \\
& +\alpha_{112}\left(p_{1}^{4} p_{2}^{2}+p_{1}^{2} p_{2}^{4}+p_{2}^{4} p_{3}^{2}+p_{2}^{2} p_{3}^{4}+p_{3}^{4} p_{1}^{2}+p_{3}^{2} p_{1}^{4}\right) \\
& +\alpha_{123} p_{1}^{2} p_{2}^{2} p_{3}^{2}-E_{1} P_{1}-E_{2} P_{2}-E_{3} P_{3}
\end{aligned}
$$

where $p_{i}$ are the orthogonal components of polarization, measured parallel to the pseudo-cubic axes of the perovskite unite cell, $E_{i}$ represents the applied electric field. According to Equation (1), the energy profiles of PZT40/60 with a rhombohedral phase were plotted under an electric field parallel and perpendicular to the [111] polar direction, as shown in Figure 11. For zero electric field, the minimum free energy exists at the polar vector along the [111] directions $\left(\left|p_{1}\right|=\left|p_{3}\right|\right)$, indicating that the rhombohedral phase is the equilibrium state. The tetragonal state with its polar vector along the [001] direction $\left(p_{1}=0\right)$ and the polarization rotation path from the $\mathrm{R}$ to $\mathrm{T}$ phase are also marked in Figure 11. (a)

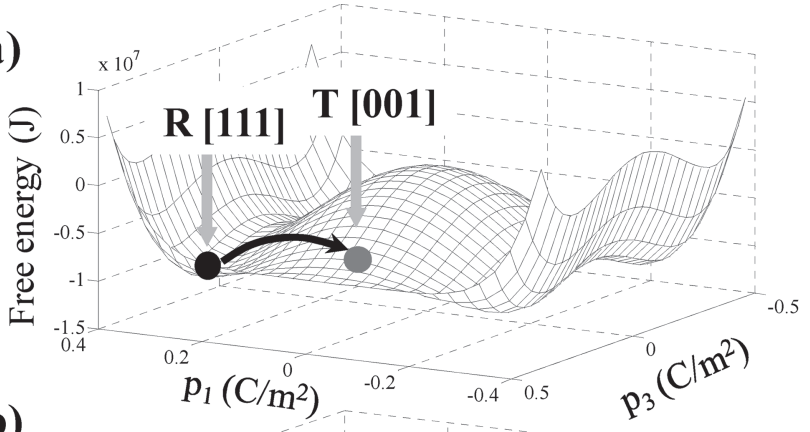

(b)

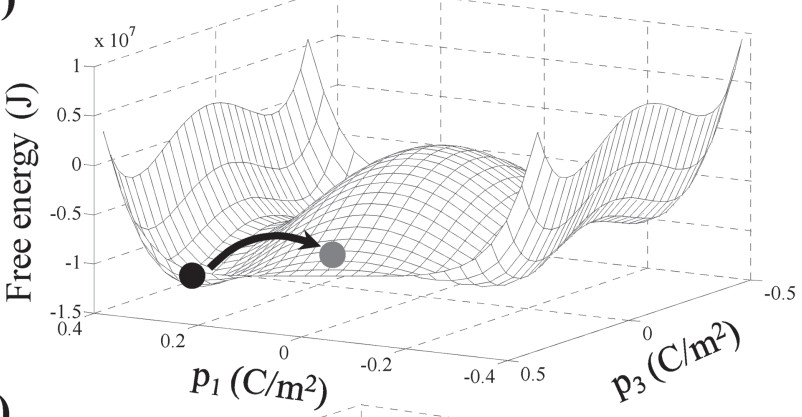

(c)

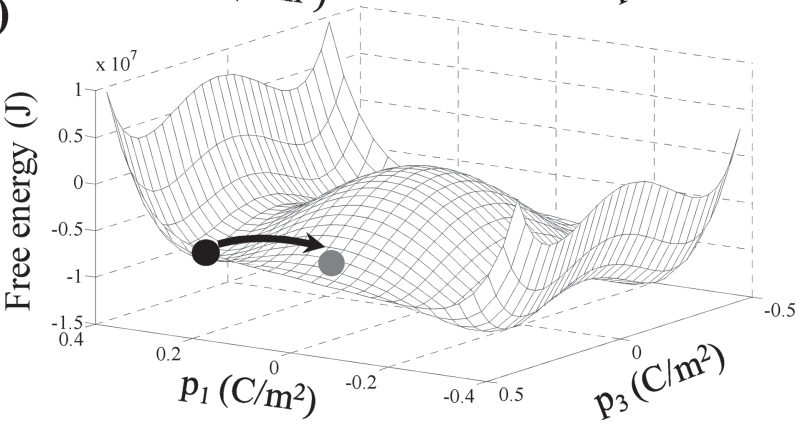

Figure 11. Gibbs free energy profile for PZT60/40 $(\mathrm{Zr} / \mathrm{Ti}=60 / 40)$ at a) zero bias field, b) $50 \mathrm{kV} \mathrm{cm}^{-1}$ along the [111] direction (parallel electric field), c) $50 \mathrm{kV} \mathrm{cm}^{-1}$ along the [-1-12] direction (perpendicular electric field). Under the above conditions, the polarization $p_{x}$ and $p_{y}$ are equivalent for the free energy of PZT60/40. Thus, the free energies are plotted with respect to the polar vectors $p_{1}$ and $p_{3}$, where $p_{x}=p_{y}=p_{1}, p_{z}=p_{3}$. The coefficients in Equation 1 have been previously reported. ${ }^{[43]}$ 




(a)

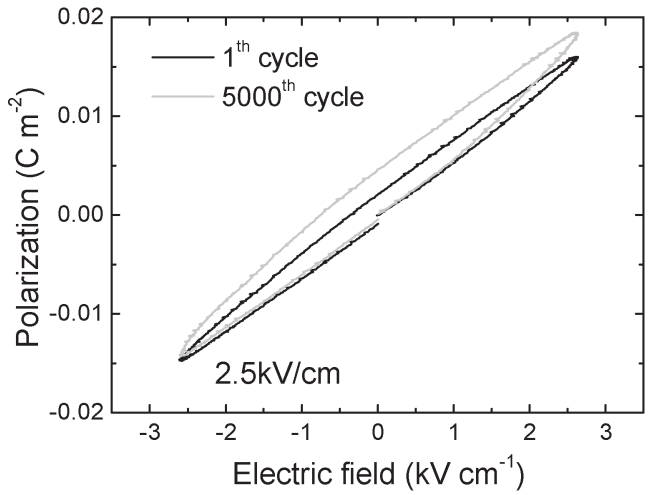

(c)



(b)

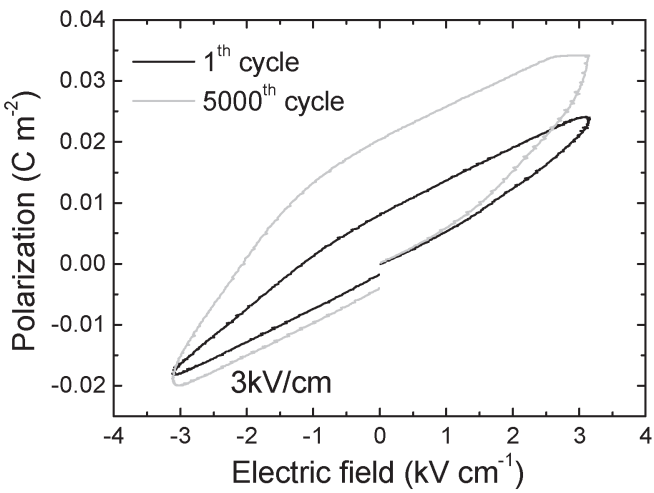

(d)

Figure 12. The polarization versus electric field behavior for $[111]_{C}$ poled rhombohedral PIN-PMN-PT crystals under various levels of electric fields, where the ac field was applied to the $(-110)_{C}$ faces.

When applying the electric field along the [111] polar direction, it is observed that the rhombohedral phase becomes more stable because of the lower free energy, which makes the polarization rotation harder, as shown in Figure 11b. Based on this notion, the observed reduction of shear piezoelectric coefficient with increasing electric field along the [111] direction in rhombohedral PZN-PT crystals can be explained. ${ }^{[36]}$ On the other hand, when the electric field is applied perpendicular to the polar direction (along [-1-12]), as shown in Figure 11c, the free energy of the rhombohedral phase (polar vector along [111] direction) increases and the polarization rotation path from the $\mathrm{R}$ to $\mathrm{T}$ phase flattens, indicating an increase in shear piezoelectric response. Thus, an enhanced shear piezoelectric coefficient can be expected when applying a perpendicular dc electric field.

\subsection{AC Electric Field Stability for Shear Piezoelectric Response}

The ac-field stability of relaxor-PT based crystals is analyzed in the following. Figure 12 shows the polarizationelectric field (PE) loops for rhombohedral PIN-PMN-PT crystals as a function of the electric field. The small-signal impedance characteristics for a thickness shear vibration mode, as a function of the ac drive field after 5000 cycles, are shown in Figure 13. At an electric field below $\mathrm{cm}^{-1}$, which is half of the coercive field $\left(E_{C}\right.$ about $5 \mathrm{kV} \mathrm{cm}^{-1}$ ), the PE loop after 5000 cycles was found to have the same shape as the loop after the first cycle, exhibiting no domain reversal or fatigue in the crystals. The field stability

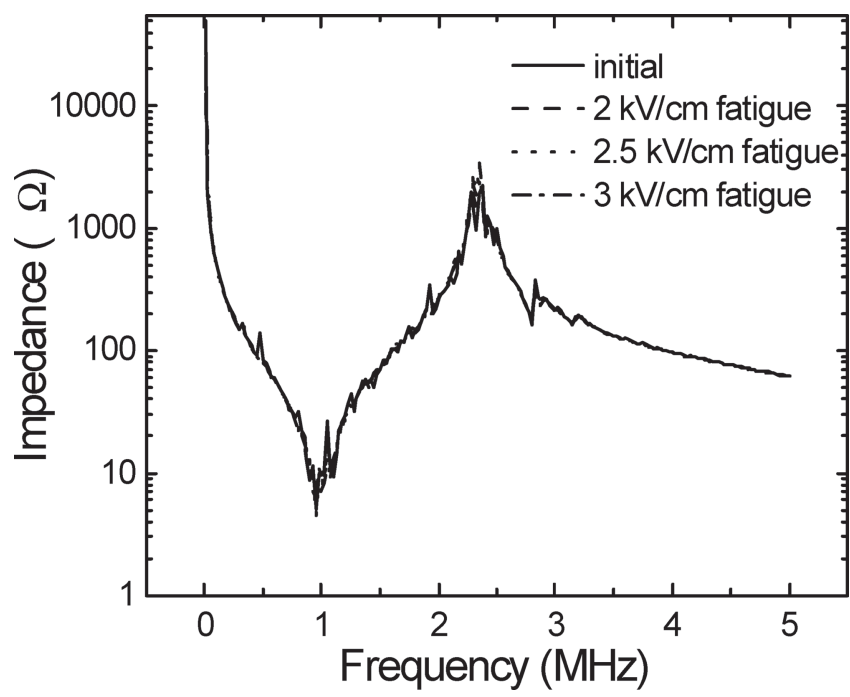

Figure 13. The impedance spectra of PIN-PMN-PT crystals in shear vibration mode; the impedance spectra were measured after 5000 cycles at various levels of ac field. 
Table 3. AC-field stability and related properties for PIN-PMN-PT crystals. Data reproduced with permission. ${ }^{[62]}$ Copyright 2011, IEEE.

\begin{tabular}{|c|c|c|c|c|c|c|c|c|c|c|c|}
\hline & Domain configuration & Material & $E_{\mathrm{C}}\left[\mathrm{kV} \mathrm{cm}^{-1}\right]$ & $E_{\text {init }}\left[\mathrm{kV} \mathrm{cm}^{-1}\right]$ & $\varepsilon$ & $s^{D}\left[\mathrm{pm}^{2} \mathrm{~N}^{-1}\right]$ & $d_{15}\left[\mathrm{pC} \mathrm{N} \mathrm{N}^{-1}\right]$ & $k_{15}$ & $\mathrm{Nr}[\mathrm{Hz} \mathrm{m}]$ & ac-field stability $\left[\mathrm{kV} \mathrm{cm}^{-1}\right]$ & $Q$ \\
\hline$[111]^{a)} /(1-10)^{b}$ & $1 R$ & Pure PIN & 4.5 & 0 & 6000 & 27 & 3500 & 0.93 & 470 & 2 & 30 \\
\hline \multirow[t]{2}{*}[011]{$\left.^{\mathrm{a})} /(0-11)^{\mathrm{b}}\right)$} & 10 & Pure PIN & 5.5 & 0 & 5600 & 27 & 3400 & 0.95 & 380 & 2 & 20 \\
\hline & & Mn-PIN & 8.9 & 0.6 & 5800 & 27 & 3500 & 0.95 & 360 & 5 & 25 \\
\hline \multirow[t]{2}{*}[001]{$^{\mathrm{a})} /(100)^{\mathrm{b})}$} & $1 \mathrm{~T}$ & Pure PIN & 11.0 & 0 & 15000 & 14 & 2200 & 0.85 & 850 & 4 & 20 \\
\hline & & Mn-PIN & 11.5 & 1.5 & 8000 & 14 & 1200 & 0.77 & 950 & 6 & 33 \\
\hline
\end{tabular}

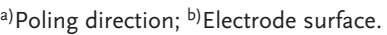

( $2 \mathrm{kV} \mathrm{cm}^{-1}$ ) was also confirmed by the impedance-frequency characteristics, as no change was observed in impedance. When further increasing the ac-field level, the PE loops became nonlinear and exhibited hysteresis behavior, meanwhile the anti-resonance frequency was found to shift to a lower frequency range, demonstrating the instability of the domain configuration at ac electric fields higher than $2 \mathrm{kV}$ $\mathrm{cm}^{-1}$, limiting the crystal usage range to $<1 / 2 E_{\mathrm{C}}$.

To improve the field stability of relaxor-PT based crystals, two approaches have been proposed. The first approach is to use tetragonal relaxor-PT crystals with improved coercive field (ca. 9-11 $\mathrm{kV} \mathrm{cm}^{-1}$ ); the other one is to use 'acceptor' modified crystals, where the internal bias field stabilizes the polarization. ${ }^{60,61]}$ Both pure and Mn-modified PIN-PMN-PT crystals, with rhombohedral, orthorhombic, and tetragonal phases were investigated. ${ }^{[62]}$ The general properties and ac electric field stability of the PIN-PMN-PT crystals are listed in Table 3. As expected, the ac-field stability can be enhanced by both approaches. Of particular interest is that for the Mn-modified orthorhombic crystals, both high electric-field drive stability $\left(5 \mathrm{kV} \mathrm{cm}^{-1}\right)$ and piezoelectric coefficient $\left(3500 \mathrm{pC} \mathrm{N}^{-1}\right)$ were obtained.

\section{Mechanical Quality Factor Q for Shear Vibration Modes}

The mechanical quality factor $Q$ is an important parameter for high-power applications. Thus, the shear-mode quality factors for various single-domain PIN-PMN-PT crystals were investigated, the results are listed in Table 3. As presented, all quality factors were found to be on the order of 20-30. For comparison, low quality factors for the longitudinal mode in domain-engineered crystals on the order of 100-300 have been found previously. ${ }^{[49,63]}$ However, high longitudinal mechanical quality factors, being on the order of $>2000$, were found in the $[111]_{\mathrm{C}}$ poled single-domain rhombohedral ${ }^{[63]}$ and $[001]_{\mathrm{C}}$ poled single-domain tetragonal crystals. ${ }^{[64]}$ Based on these results, it is proposed that the high level of mechanical losses (low mechanical quality Q) are associated with the polarization rotation process, including the shear mode of single-domain crystals and longitudinal mode of domain-engineered crystals. High quality factors were obtained in longitudinal mode single-domain crystals because of the absence of polarization rotation processes.

In contrast to the piezoelectric coefficient, the mechanical quality factor $Q$ is more sensitive to other parameters, such as the test frequency, electric-field level, and preload stress. ${ }^{[65]}$ Of particular interest is that both a high level of mechanical quality factor and piezoelectric coefficient were found in the face shear vibration mode (36-mode) of [011 $]_{C}$ poled rhombohedral crystals, ${ }^{[66]}$ whereby the high piezoelectric activity is associated with the polarization rotation process, as shown in Figure 14. For 36-mode crystals, the applied working electric field is parallel to the poling direction, whereas the working electric field is perpendicular to the poling direction for thickness shear crystals (15-mode, 24-mode). Thus, the mechanical quality factor may also be related to the polarization rotation angle, which is under investigation.

\section{Conclusion}

The piezoelectric behavior in relaxor-PT based crystals with rhombohedral, orthorhombic, and tetragonal phases, was investigated. High shear piezoelectric coefficients were generally obtained in single-domain rhombohedral, orthorhombic, and tetragonal crystals with compositions near the MPBs. The high shear piezoelectric activity was a critical characteristic for relaxor-PT based crystals, and, as a result, the longitudinal piezoelectric responses could greatly be improved in domainengineered crystals.

From an application viewpoint, interesting properties were observed for orthorhombic and tetragonal crystals compared to their rhombohedral counterparts. High shear piezoelectric responses with improved thermal stability $\left(d_{24}\right)$ and ac drive

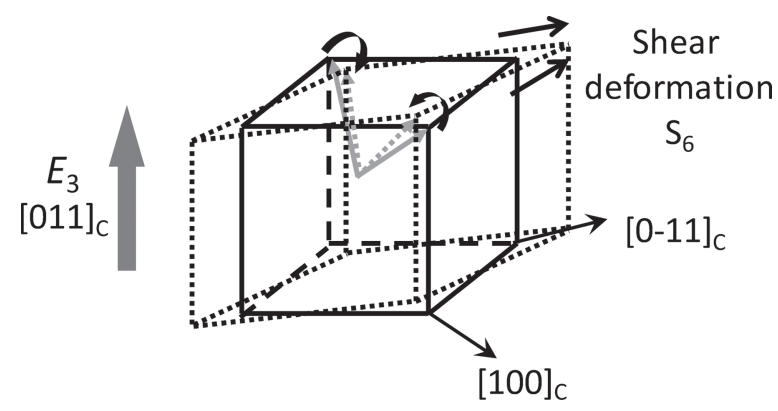

Figure 14. Schematic polarization rotation process and related face shear piezoelectric deformation for the 36-mode, where the gray arrows represent the spontaneous polar vectors $[-111]$ and [111] of rhombohedral crystals. 
(a)

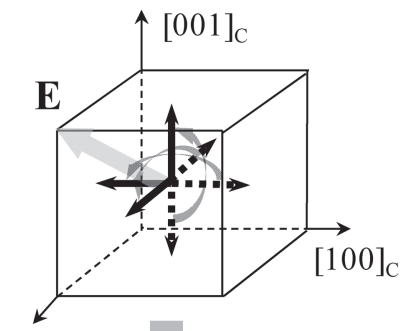

$[010]_{\mathrm{C}}$
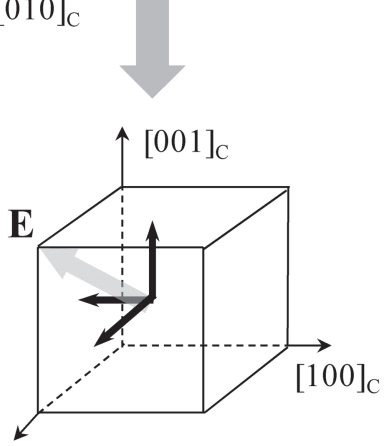

$[010]_{\mathrm{C}}$ (b)



$[010]_{\mathrm{C}}$

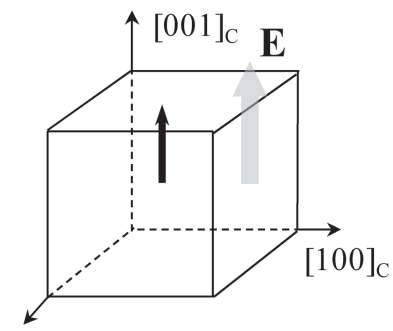

$[010]_{\mathrm{C}}$
Figure 15. Domain evolution of tetragonal crystals by poling along a) the [111] direction and b) the [001] direction.

field stability ( $d_{15}$, Mn-modified crystals) were obtained in orthorhombic crystals. Moreover, a high electromechanical coupling $k_{33}(>84 \%)$ and mechanical quality factor $Q(>2000)$ were concurrently obtained in tetragonal relaxor-PT crystals, with broadened temperature usage range close to their respective $T_{\mathrm{C}}$.

\section{Experimental Section}

Poling Process: As demonstrated in Section 2, relaxor-PT crystals were poled along their non-polar directions to obtain domain-engineered structures for high longitudinal piezoelectric responses, whereas the crystals were poled along their polar directions in order to achieve high shear piezoelectric responses in single-domain structures.

In contrast to domain-engineered crystals, such as [001] poled rhombohedral crystals (4R) and [111] poled tetragonal crystals (3T), single-domain crystals were found to crack easily during the poling process, thus, limiting their applications.

To design a crack-free poling method for single-domain crystals, the domain evolution of tetragonal crystals was analyzed by poling along the [001] polar direction and [111] non-polar directions, respectively.

For tetragonal ferroelectric crystals, six different domains, namely along the [001], [010], [100], [-100], [0-10], and [00-1] directions, coexist prior to the poling process. As the crystals were poled along the [111] direction, domains along the [-100], [0-10], and [00-1] directions transformed to the other three electrically favored domains, forming engineered domain configuration ' $3 \mathrm{~T}$ ', as shown in Figure 15a. During the poling process, the electric-field-induced strain by domain switching was minimal, because the spontaneous strain of these six domains were equivalent with respect to the [111] direction.

On the other hand, if the crystals were poled along the [001] direction, domains along the [010], [100], [-100], [0-10], and [00-1] directions transformed to the electrically favored [001] domain, leading to the single-domain state ' $1 \mathrm{~T}$ ', as shown in Figure 15b. During this poling process, the electric-field-induced strain was significantly large and abrupt, because of the $90^{\circ}$ ferroelectric/ferroelastic domain switching from the [010], [100], [-100], or [0-10] domains to the [001] domain. As expected, the strain was found to be $>0.6 \%$, with a sharp strain-electric field behavior around the coercive field, for [001]-oriented tetragonal PIN-PMN-PT crystals. ${ }^{[3]}$

According to the above analysis, the crack phenomenon of singledomain crystals was attributed to the large strain variation, which was induced by the non- $180^{\circ}\left(90^{\circ}\right)$ ferroelectric/ferroelastic domain switch. Therefore, in order to avoid cracking, it is necessary to minimize the strain variation during the poling process. Based on this notion, a special poling approach was used to obtain single-domain crystals with various ferroelectric phases, as described in the following.

The tetragonal relaxor-PT crystals were poled along the [001] direction above the Curie temperature, and then slowly cooled down to room temperature under the same electric field. By this poling method, only one domain along the [001] direction was retained with decreasing temperature from the paraelectric to the ferroelectric phase. Compared to the room-temperature poling process, a $90^{\circ}$ domain switch was avoided in the above poling process.

For rhombohedral and orthorhombic crystals, the poling electric field was applied along the [111] and [011] directions, respectively. The crystals were also poled above the Curie temperature, whereby the poling electric field was larger than the tetragonal-rhombohedral/orthorhombic phase transition electric field, in order to guarantee a single-domain state with its polar vector along the [111]/[011] direction for rhombohedral/ orthorhombic crystals in the temperature range of $T_{\mathrm{C}}$ to $T_{\mathrm{RT}} / T_{\mathrm{OT}}$.

Electrical Measurements: After the polarization process, the electrodes were removed and attached to large surfaces. The dielectric permittivity was determined at $1 \mathrm{kHz}$, using an HP4284A multi-frequency LCR meter. The resonance and antiresonance frequencies were measured using an HP4294A impedance-phase gain analyzer. The shear piezoelectric coefficients and electromechanical couplings were determined by resonance and antiresonance frequencies, following the IEEE standards on piezoelectricity. The mechanical quality factor $Q$ was calculated based on the Butterworth-Van Dyke equivalent circuit. High-field polarization behaviors were determined using a modified Sawyer-Tower circuit, with a measuring frequency of $10 \mathrm{~Hz}$, which is much higher than the domain reversal frequency.

\section{Acknowledgements}

The authors from Xi'an Jiaotong University acknowledge the National Basic Research Program of China (973 Program) under Grant No. 2009CB623306, International Science \& Technology Cooperation Program of China under Grant No. 2010DFR50480, National Nature Science Foundation of China (Grant Nos. 10976022 and 50632030). The authors thank Dr. J. Luo from TRS Technologies, Inc. for offering single crystals. The work was supported by the NIH under Grant No. P41-EB21820 and ONR.

Received: December 23, 2010 Published online: April 20, 2011

[1] S.-E. Park, T. R. Shrout, J. Appl. Phys. 1997, 82, 1804

[2] S. E. Park, T. R. Shrout, Mater. Res. Innovations 1997, 1, 20.

[3] S. J. Zhang, T. R. Shrout, IEEE Trans. Ultrasonics, Ferroelectrics, Frequency Control 2010, 57, 2138.

[4] H. S. Luo, G. S. Xu, H. Q. Xu, P. C. Wang, Z. W. Yin, Jpn. J. Appl. Phys. 2000, 39, 5581.

[5] J. Kuwata, K. Uchino, S. Nomura, Jpn. J. Appl. Phys. 1982, 21, 1298.

[6] S. J. Zhang, C. A. Randall, T. R. Shrout, IEEE Trans. Ultrasonics, Ferroelectrics, Frequency Control 2005, 52, 564. 
[7] Fei Li, S. Zhang, Z. Xu, X. Wei, J. Luo, T. R. Shrout, J. Appl. Phys. 2010, 108, 034106

[8] H. Fu, R. E. Cohen, Nature 2000, 403, 281.

[9] L. Bellaiche, A. Garcia, D. Vanderbilt, Phys. Rev. B 2001, 64, 060103.

[10] B. Noheda, Curr. Opin. Solid State Mater. Sci. 2002, 6, 27.

[11] B. Noheda, D. E. Cox, G. Shirane, J. A. Gonzalo, L. E. Cross, S.-E. Park, Appl. Phys. Lett. 1999, 74, 2059.

[12] R. Guo, L. E. Cross, S-E. Park, B. Noheda, D. E. Cox, G. Shirane, Phys. Rev. Lett. 2000, 84, 5423.

[13] G. S. Xu, H. S. Luo, H. Q. Xu, Z. W. Yin, Phys. Rev. B 2001, 64, $020102(R)$.

[14] B. Noheda, D. E. Cox, G. Shirane, J. Gao, Z.-G. Ye, Phys. Rev. B 2002, 66, 054104.

[15] D. La-Orauttapong, B. Noheda, Z.-G. Ye, P. M. Gehring, J. Toulouse, D. E. Cox, G. Shirane, Phys. Rev. B 2002, 65, 144101.

[16] D. Vanderbilt, M. H. Cohen, Phys. Rev. B 2001, 63, 094108.

[17] B. Noheda, D. E. Cox, G. Shirane, S.-E. Park, L. E. Cross, Z. Zhong, Phys. Rev. Lett. 2001, 86, 3891.

[18] L. Bellaiche, A. Garcia, D. Vanderbilt, Phys. Rev. B. 2001, 64, 060103.

[19] D. Damjanovic, M. Budimir, M. Davis, N. Setter, Appl. Phys. Lett. 2003, 83, 527.

[20] M. Budimir, D. Damjanovic, N. Setter, J. Appl. Phys. 2003, 94, 6753.

[21] M. Budimir, D. Damjanovic, N. Setter, Phys. Rev. B 2005, 72, 064107.

[22] M. Davis, M. Budimir, D. Damjanovic, N. Setter, J. Appl. Phys. 2007, 101, 054112

[23] D. Damjanovic, IEEE Trans. Ultrasonics, Ferroelectrics, Frequency Control 2009, 56, 1574.

[24] P. Han, J. Tian, presented at "US Navy Workshop on Acoustic Transduction Materials and Devices", The Penn State Conference Center, State College, PA, May, 2006.

[25] J. Luo, S. J. Zhang, W. Hackenberger, T. R. Shrout, presented at "US Navy Workshop on Acoustic Transduction Materials and Devices", The Penn State Conference Center, State College, PA, May, 2010.

[26] K. Echizenya, M. Matsushita, Y. Tachi, T. Addona, presented at "US Navy Workshop on Acoustic Transduction Materials and Devices", The Penn State Conference Center, State College, PA, May, 2010.

[27] Y. Hosono, Y. Yamashita, H. Sakamoto, N. Ichinose, Jpn. J. Appl. Phys. Part 1 2003, 42, 5681.

[28] J. Tian, P. D. Han, X. L. Huang, H. X. Pan, Appl. Phys. Lett. 2007, 91, 222903.

[29] S. J. Zhang, J. Luo, W. Hackenberger, T. R. Shrout, J. Appl. Phys. 2008, 104, 064106

[30] S. J. Zhang, J. Juo, W. Hackengerger, N. Sherlock, R. J. Meyer Jr, T. R. Shrout, J. Appl. Phys. 2009, 105, 104506.

[31] G. Xu, K. Chen, D. Yang, J. Li, Appl. Phys. Lett. 2007, 90, 032901.

[32] L. H. Liu, X. Wu, X. Y. Zhao, X. Q. Feng, W. P. Jing, H. S. Luo, IEEE Trans. Ultrasonics, Ferroelectrics, Frequency Control 010, 57, 2154.

[33] F. Li, S.J. Zhang, Z. Xu, X Wei, J. Luo, T. R. Shrout, J. Appl. Phys. 2010, 107, 054107

[34] S. J. Zhang, L. Lebrun, S. F. Liu, S. Rhee, C. A. Randall, T. R. Shrout, Jpn. J. Appl. Phys. 2002, 41, L1099.

[35] S. J. Zhang, L. Lebrun, S. Rhee, C. A. Randall, T. R. Shrout, Appl. Phys. Lett. 2002, 81, 892.

[36] S.F. Liu, W. Ren, B. K. Mukherjee, S. J. Zhang, T. R. Shrout, P. W. Rehrig, W. S. Hackenberger, Appl. Phys. Lett. 2003, 83, 2886.
[37] J. Jin, K. K. Rajan, L. Lim, IEEE Trans. Ultrasonics, Ferroelectrics, Frequency Control 2007, 54, 2222.

[38] P. D. Han, W. L. Yan, J. Tian, X. L. Huang, H. X. Pan, Appl. Phys. Lett. 2005, 86, 052902.

[39] R. Zhang, B. Jiang, W. Cao, Appl. Phys. Lett. 2003, 82, 3737.

[40] F. Li, S. Zhang, Z. Xu, X Wei, J. Luo, T. R. Shrout, Appl. Phys. Lett. 2010, 97, 252903

[41] M. Davis, D. Damjanovic, D. Hayem, N. Setter, J. Appl. Phys. 2005, 98, 014102.

[42] M. E. Lines, A. M. Glass, Principles and Applications of Ferroelectrics and Related Materials, Clarendon, Oxford, UK, 1979.

[43] M. J. Haun, E. S. Furman, J. Jang, L. E. Cross, Ferroelectrics 1989, 99, 13.

[44] X. Z. Liu, S. J. Zhang, J. Luo, T. R. Shrout, W. W. Cao, J. Appl. Phys. 2009, 106, 074112.

[45] R. Zhang, B. Jiang, W. Cao, J. Appl. Phys. 2001, 90, 3471.

[46] R. Zhang, B. Jiang, W. Jiang, W. Cao, Appl. Phys. Lett. 2006, 89, 242908.

[47] E. Sun, S. Zhang, J. Luo, T. R. Shrout, W. Cao, Appl. Phys. Lett. 2010, 97, 032902.

[48] S. J. Zhang, F. Li, J. Luo, R. Xia, W. Hackenberger, T. R. Shrout, Appl. Phys. Lett. 2010, 97, 132903.

[49] Fei Li, S. Zhang, Z. Xu, X. Wei, J. Luo, T. R. Shrout, J. Am. Ceram. Soc. 2010, 93, 2731

[50] X. Liu, S. J. Zhang, J. Luo, T. R. Shrout, W. Cao, Appl. Phys. Lett. 2010, 96, 012907 .

[51] H. Cao, V. H. Schmidt, R. Zhang, W. Cao, H. Luo, J. Appl. Phys. 2004, $96,549$.

[52] S. J. Zhang, L. Lebrun, C. A. Randall, T. R. Shrout, Phys. Status Solidi A 2005, 202, 151.

[53] T. Yamada, J. Appl. Phys. 1972, 43, 328.

[54] D. Damjanovic, Phys. Rev. B 1997, 55, R649.

[55] F. Li, S. J. Zhang, D. B. Lin, J. Luo, X. Zhuo, X. Y. Wei, T. R. Shrout, J. Appl. Phys. 2011, 109, 014108.

[56] M. Davis, D. Damjanovic, N. Setter, Phys. Rev. B 2006, 73, 014115.

[57] F. Li, S. J. Zhang, Z. Xu, X. Wei, J. Luo, T. R. Shrout, Appl. Phys. Lett. 2010, 96, 192903.

[58] D. S. Paik, S. E. Park, T. R. Shrout, J. Mater. Sci. 1999, 34, 469.

[59] X. Jiang, P. W. Rehrig, W. S. Hackenberger, T. R. Shrout, AIP Conf. Proc. 2006, 823, 1783

[60] B. Jaffe, W. R. Cook, H. Jaffe, Piezoelectric Ceramics, Academic Press, New York, NY 1971

[61] M. A. Hentati, M. Guennou, H. Dammak, H. Khemakhem, M. P. Thi, J. Appl. Phys. 2010, 107, 064108.

[62] S. J. Zhang, F. Li, J. Luo, R. Xia, W. Hackenberger, T. R. Shrout, IEEE Trans. Ultrasonics, Ferroelectrics, Frequency Control 2011, $58,274$.

[63] S. J. Zhang, N. P. Sherlock, R. J. Meyer, Jr., T. R. Shrout, Appl. Phys. Lett. 2009, 94, 162906.

[64] F. Li, S. Zhang, J. Luo, W. Hackenberger, T. R. Shrout, presented at "US Navy Workshop on Acoustic Transduction Materials and Devices", The Penn State Conference Center, State College, PA, May, 2010.

[65] N. P. Sherlock, S. J. Zhang, J. Luo, H. Y. Lee, T. R. Shrout, R.J. Meyer, Jr, J. Appl. Phys. 2010, 107, 074108.

[66] R. J. Meyer, Jr., T. M. Tremper, D. C. Markley, D. Van Tol, P. Han, J. Tian, presented at "US Navy Workshop on Acoustic Transduction Materials and Devices", Penn State Conference Center, State College, PA, May, 2010. 